\title{
Sound radiation from composite plates
}

H Aygun London South Bank University, School of the Built Environment and Architecture, 103 Borough Rd, London, SE1 OAA, UK. aygunh@lsbu.ac.uk

\section{Introduction}

The vibrations of the structures that are made of isotropic and homogeneous materials has been an important subject in the study of noise control, in the aeronautical industry, in the study of fluid-solid interactions and in construction industry for double leaf partitions as building structures. When a sound wave impinges on a structure, a part of acoustic energy is reflected back into source medium, a part is dissipated in the structure and the rest of acoustic energy is transmitted through the structure into the other medium. In building acoustics applications, the reflected acoustic energy builds up a reverberant sound field in the source room that in turn vibrates the walls. The vibrations in the common walls radiate sound directly into the receiver room. The vibrations in the other walls of the source room travel as structure-borne noise to the all walls of the receiving room and radiate sound into the receiver room.

Complex structures may be modelled as systems that are made of individual plate like elements. The theory of vibration of porous and non-porous structures is a well-known branch of engineering mechanics. Previous works on classical theory of the plate [1-7] has investigated vibration of isotropic and anisotropic plates for various boundary conditions. The vibration of porous plates can be described using two coupled equations [8], which are based on Biot's stress-strain relations [9, 10] and which introduce two types of compressional waves ('fast' and 'slow') and a shear wave. They assumed that the thickness of plate is smaller than the wavelength and that interaction can take place between the slow waves and the bending waves in the plate. They also ignored the amplitude of the fast wave. Galerkin's variational techniques were applied to porous plates [11-13], taking into account a classical set of trial functions obtained from the linear combination of trigonometric and hyperbolic functions for various boundary conditions. The effects of fluid loading on the vibration of rectangular porous plates and on their radiated sound power was investigated by including an extra term into the equations for the porous plate vibration, corresponding to the additional external force acting on the plate [14]. Previous study on low frequency vibration of porous plates [15] has demonstrated the existence of low frequency absorption coefficient resonance in configurations consisting of clamped poroelastic plates with an air cavity between the plates and a rigid termination. An analytical model that takes into account the effect of perforations and the effect of the flexural vibrations in the plates has been formulated and used to calculate the insertion loss in the absence, and in the presence of air flow [16, 17].

Important progress in predicting acoustic radiation from baffled structures including plates and beams has been made in the last three decades. Many previous studies have focused on the calculation of the radiation efficiency of these structures [18-21] and the radiation of sound from a baffled, rectangular plate with edges elastically restrained against deflection and rotation [22-23]. The models used for the radiation efficiency of plate-like radiators, ranged from very simple ones based on modal average expressions to refined calculations of the radiation impedance matrix with cross-modal coupling have been evaluated, and developed a new approach based on Taylor's expansion of the Green's function [24]. Variational method that is based on the Rayleight-Ritz model, can be used to determine the radiation of sound from the plates that are immersed either in a light fluid or in a heavy fluid by reducing the quadruple integral into a double integral using a specific change of variable and by integrating the double integral with a numerical method using Gaussian quadrature formulae [25-28].

Recently, Aygun and McCann [29] has studied composite recycled glass bead panels in order to assess their suitability for civil engineering application, especially in noisy urban environments, either as structural panel components that also offer acoustic insulation or as dedicated noise barriers for outdoor applications.

The aim of this paper is to investigate the vibrational and acoustical parameters of thin composite plates that are made of fibreglass, which are used for applications ranging from aerospace and automotive to construction industry. To author's best knowledge, vibroacoustical properties of composite plate have been reported in this paper for first time. The deflection of composite plate has been predicted at difference locations on the plate using the classical theory of the vibrating plate for simply supported and clamped boundary conditions. Computational simulations have been carried out to determine deformations of the plate in $3 \mathrm{D}$ for different frequency ranges for simply supported and clamped 
boundary conditions. Here, vibroacoustic indicators including the radiation efficiency, the mean square velocity, and the radiated sound power have been computed for a square composite plate. Furthermore, the radiation impedance matrix has been predicted for simply supported boundary condition by using equations for eigenfunctions and Green's function without interpolation, convergence and without reducing the quadruple integral into a double integral.

\section{Theory of plate vibration}

Wave motion in solid structures stores energy in shear as well as in compression. Many different types of waves can be seen in solid structures when a solid is excited by different ways of stressing. The solid structures should have energy storage capability in the form of potential and kinetic energy in order to allow wave propagation through the medium. Potential energy is stored in solid structure parts that have undergone elastic deformation while kinetic energy is stored in any part of the medium that has mass and is in motion. When a flat plate is subjected to a transverse, time dependent force density $F(x, y, t)$, the transverse deflection of the plate is governed by the fourth order differential equation. The transverse vibration under free wave conditions stems entirely from inertial loading. A thin, baffled square plate of dimension $a x a$ (along aces $x$ and $y$, respectively) and uniform thickness $h$ is considered in this study. The plate displacement induced by bending waves is in the direction of $z$ axis and is function of time. The geometry of the plate is shown in Figure 1.

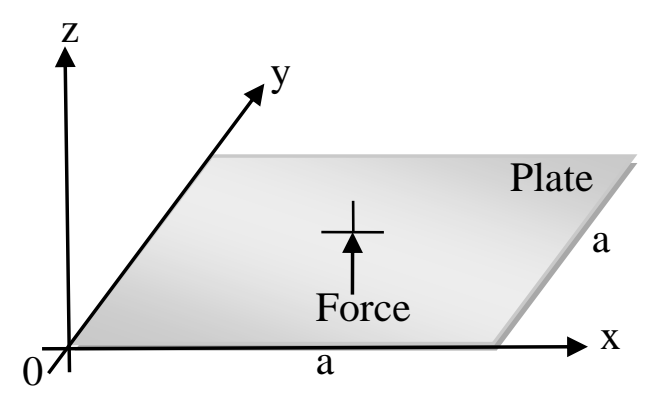

Figure 1: The geometry of a baffled plate.

The flexural wave equation for composite thin plate are given below;

$$
D \nabla^{4} w_{s}+\rho_{s} h \ddot{w_{s}}=\left\{\begin{array}{l}
0, \text { free vibration } \\
F(x, y), \text { forced vibration }
\end{array}\right\}
$$

where $w_{s}$ is the transverse plate deflection, $\ddot{w}_{s}$ is the second order derivative of the plate deflection, $D=E h^{3} / 12\left(1-v^{2}\right)$ is the flexural rigidity, $\nabla^{4}=\nabla^{2}\left(\nabla^{2}\right)$ and $\nabla^{2}=\partial^{2} / \partial x^{2}+\partial^{2} / \partial y^{2}$ in the system of coordinates $(x, y)$ with $x$ and $y$ parallel to the plate sides of length $a$ and $b$ respectively, $\rho_{s}$ is the mass density, $E$ is the Young's modulus of the plate, and $v$ is the Poisson ratio of the plate.

The plate deflection $w_{s}$ for harmonic wave motion is expressed in the form of the beam functions as given below;

$$
w_{s}(x, y)=\sum_{m=0}^{\infty} \sum_{n=0}^{\infty} A_{m n} X_{m}(x) Y_{n}(y)
$$

where $A_{m n}$ is the unknown coefficients to be determined, $m, n=0,1,2,3 \ldots \ldots$, and $X_{m}$ and $Y_{n}$ are the beam functions in $x$ and $y$ direction respectively.

The beam functions have been selected to satisfy different boundary conditions at the edges of the plate. An appropriate trigonometric function for vibrating beams has been used for $X_{m}$ and $Y_{n}$ different 
boundary conditions. For simply supported plates, the beam functions are $X_{m}(x)=\sin (m \pi x / a)$, and $Y_{n}(y)=\sin (n \pi y / b)$ which should satisfy the equations of equilibrium. The boundary conditions for simply supported edges of the plate are $w=0, \frac{\partial^{2} w}{\partial x^{2}}=0$, for $x=0$ and $x=a$ and $w=0, \frac{\partial^{2} w}{\partial y^{2}}=0$, for $y=0$ and $y=b$. The shape of each mode of vibration of plate can be determined from Equation (2) by knowing the relative values of $A_{m n}$ and the values of $X_{m}$ and $Y_{n}$ functions. In the static and dynamic analysis, the excitation function $F(x, y)$ has been expanded into double infinite sine series of variables $x$ and $y$ for each value of the couple $(m, n)$ by using the equation below;

$$
F(x, y)=\sum_{m=0}^{\infty} \sum_{n=0}^{\infty} F_{m n} \sin (m \pi x / a) \sin (n \pi y / b)
$$

where $F_{m n}$ are the expansion coefficients.

The properties of the plate used for numerical analysis are given in the Table 1.

Table 1: Properties of thin composite plate

\begin{tabular}{|l|l|l|l|l|l|l|}
\hline $\begin{array}{l}\text { Length } \\
(\mathrm{m})\end{array}$ & $\begin{array}{l}\text { Width } \\
(\mathrm{m})\end{array}$ & $\begin{array}{l}\text { Thickness } \\
(\mathrm{m})\end{array}$ & $\begin{array}{l}\text { Density } \\
\left(\mathrm{kg} / \mathrm{m}^{3}\right)\end{array}$ & $\begin{array}{l}\text { Young's } \\
\text { Modulus }(\mathrm{Pa})\end{array}$ & $\begin{array}{l}\text { Loss } \\
\text { Factor }\end{array}$ & $\begin{array}{l}\text { Poisson } \\
\text { Ratio }\end{array}$ \\
\hline 0.50 & 0.50 & 0.0025 & 1600 & $7.489 \times 10^{9}$ & 0.03 & 0.2 \\
\hline
\end{tabular}

The square velocities of thin composite plate for simply supported boundary condition are computed using a MATLAB code at the centre of the plate for $100 \mathrm{~Hz}, 500 \mathrm{~Hz}$ and $1 \mathrm{kHz}$ in Figures 2 and 3. Circular dot shows the location of the force applied to the plate. Dark red contours show the high pressure areas while dark blue contours show the minimum pressure areas.
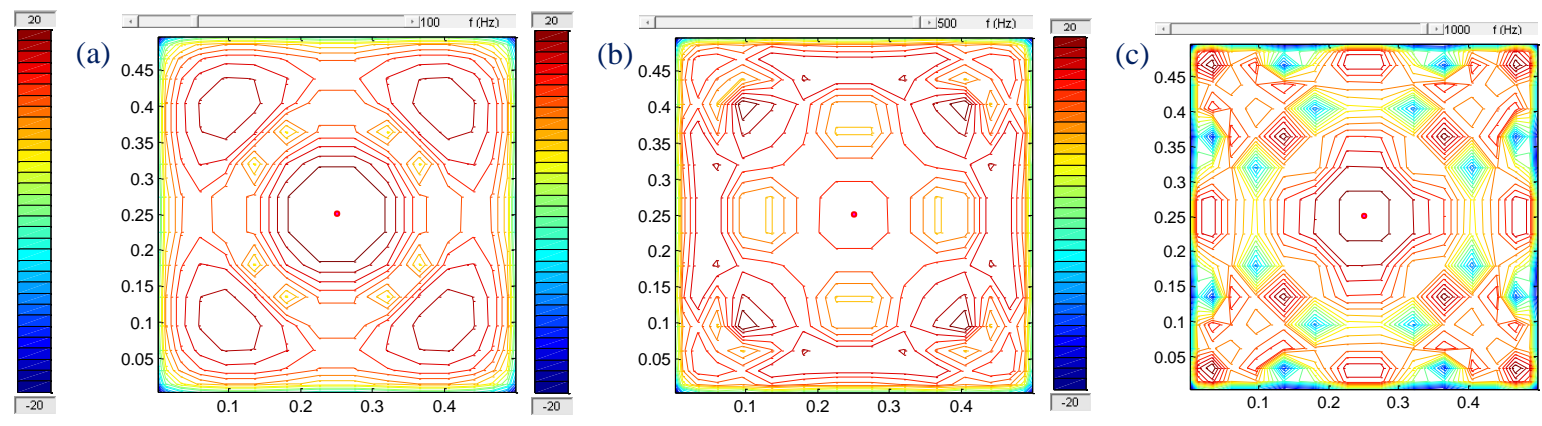

Figure 2: Square velocity (contour) of fibreglass composite simply supported plate at a) $100 \mathrm{~Hz}, \mathrm{~b}$ ) 500 $\mathrm{Hz}$, and c) $1 \mathrm{kHz}$ respectively.
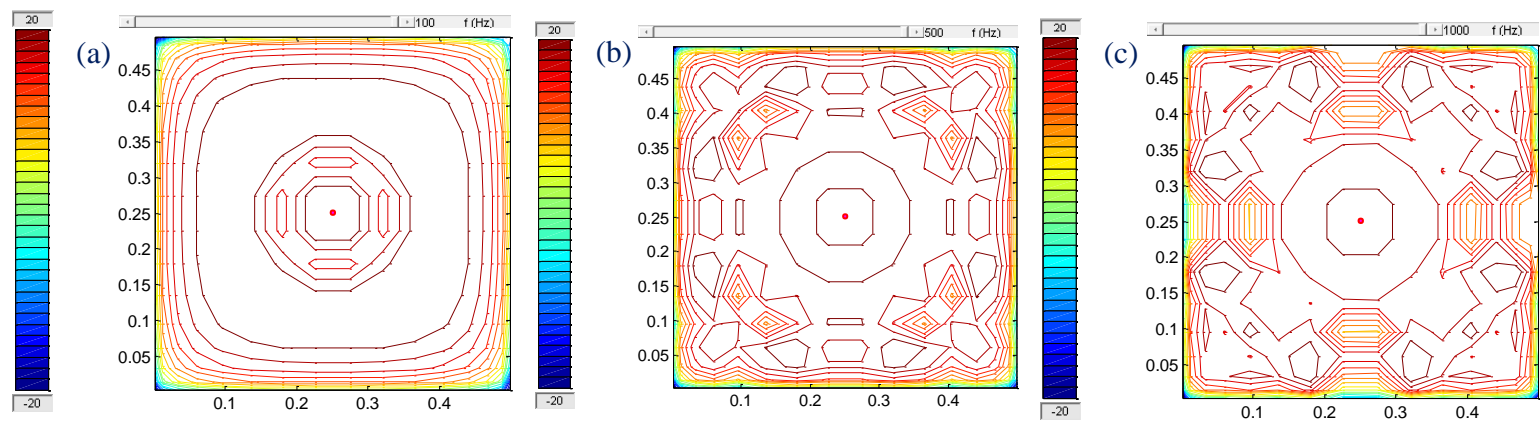

Figure 3: Square velocity (contour) of fibreglass composite clamped plate at a) $100 \mathrm{~Hz}$, b) $500 \mathrm{~Hz}$, and c) $1 \mathrm{kHz}$ respectively. 


\section{Acoustic radiation from a composite plate}

Mean square velocity is the squared velocity normal to the surface of the plate in a given frequency band and it is averaged over time and the surface area of the plate. It expresses the global behaviour of the vibration of the plate. Mean square velocity of thin composite plate is defined as a time-space average of the square vibrational velocity of plate. Mean square velocity of thin composite plate is given by [22];

$$
V^{2}=\frac{T^{-1}}{a b} \iiint_{0}^{T}\left|\frac{\partial w(x, y, t)}{\partial t}\right|^{2} d S d t
$$

where $T=\frac{2 \pi}{\omega}$, $a$ and $b$ are the coordinates of plates in $x$ and $y$ directions, respectively, and $S$ is the surface area of the plate.

The total sound field generated by vibrating plates can be expressed in terms of the distribution of sound pressure over a given surface. The radiated sound power expresses the sound energy radiated into the surrounding environment by vibration of the plate. It can be obtained from the integration of the sound intensity over the plate surface. The radiated sound power of thin composite plate is given by equation (5);

$$
W=T^{-1} \int_{0}^{T} \iint_{S} P(x, y, 0, t) \frac{\partial w(x, y, t)}{\partial t} d S d t
$$

where $P(x, y, 0, t)$ is the surface acoustic pressure which is often called Rayleigh integral, and is given by the Equation (6),

$$
P(x, y, 0)=-\rho \omega^{2} \iint_{S} w\left(x^{\prime}, y^{\prime}\right) G\left(x, y, 0 ; x^{\prime}, y^{\prime}, 0\right) d S^{\prime}
$$

where $G\left(x, y, 0 ; x^{\prime}, y^{\prime}, 0\right)$ is the Green function and it is given by the Equation (7);

$$
G\left(x, y, 0 ; x^{\prime}, y^{\prime}, 0\right)=\frac{e^{-j k R}}{2 \pi R}
$$

where $k$ is the wave number, and $R$ is the distance between points, and is given by the Equation (8);

$$
R=\sqrt{(x-y)^{2}+\left(x^{\prime}-y^{\prime}\right)^{2}},
$$

The size and shape of the vibrating plate play an important role to determine the radiation efficiency of the plate. The radiation efficiency of the plate is small at low frequencies because of larger wavelength while the radiation efficiency approaches unity at higher frequencies because of smaller wavelength of sound. The radiation efficiency expresses the ratio of the vibration energy (mean square velocity) transformed into acoustic energy (sound power). The radiation efficiency of the plate does not depend on the amplitude of vibrating structure. It can be obtained using analytical methods. The radiation efficiency of the plate is given by the Equation (9);

$$
\eta=\frac{W}{Z a b V^{2}}
$$

Where $Z$ is the characteristic impedance of air.

If the plate deflection given by Equation (2) is solved, then the mean square velocity of the plate can easily be predicted. A Gaussian quadrature scheme with 20 terms of the Legendre polynomial has been used to expand the deflection of the plate and mean square velocity of the plate with 30 elements in each direction $(m, n)$. The mean square velocity of composite plate is shown in Figure 4 while the predicted real part of the radiated sound power of a composite plate is shown in Figure 5. The radiation efficiency of the plate has been calculated by using the radiated sound power and the mean square 
velocity of the plate. The radiation efficiency of a square simply supported composite plate is shown in Figure 6.

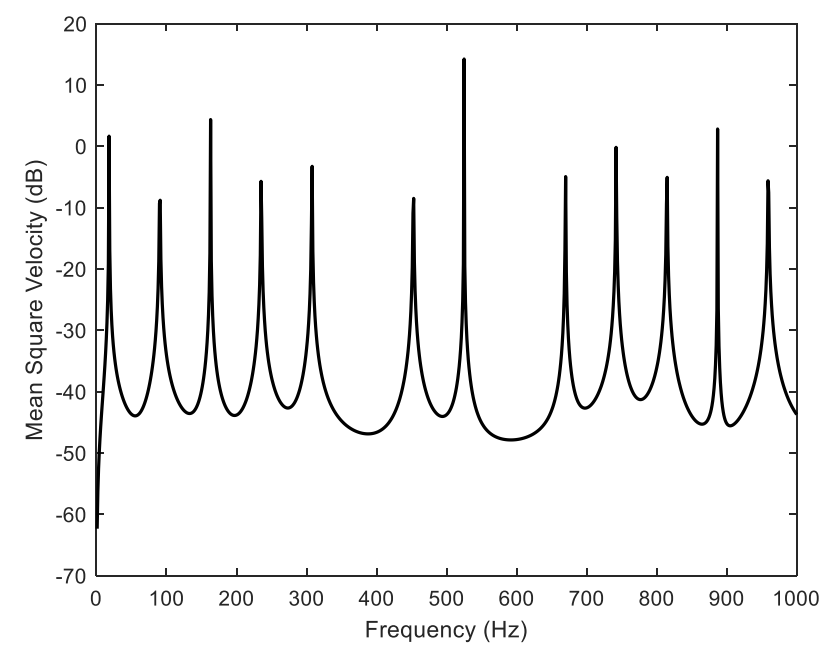

Figure 4: Mean square velocities of the composite plate at $x=0.25 \mathrm{~m}$ and $y=0.25 \mathrm{~m}$

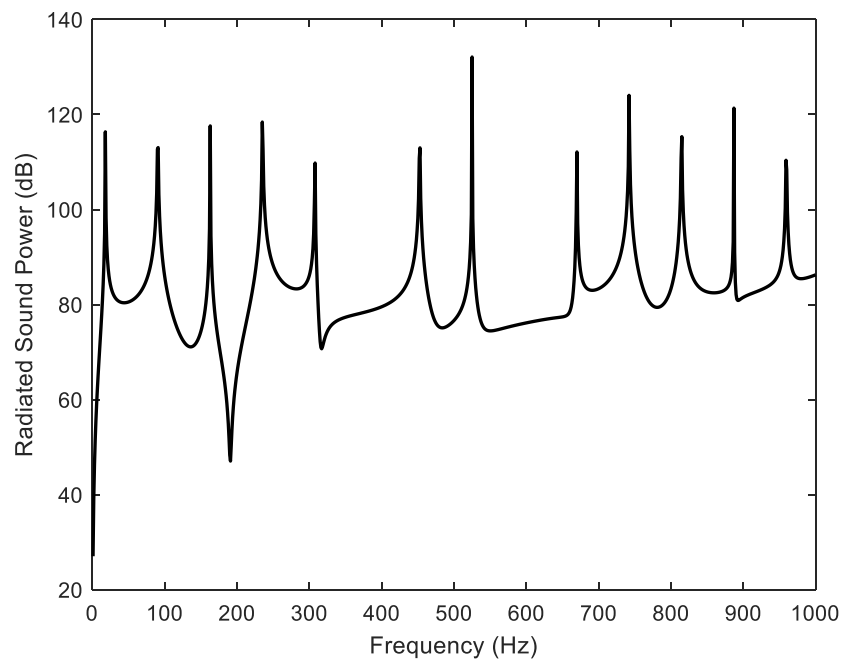

Figure 5: The real part of radiated sound power of the composite plate at $x=0.25 \mathrm{~m}$ and $y=0.25 \mathrm{~m}$

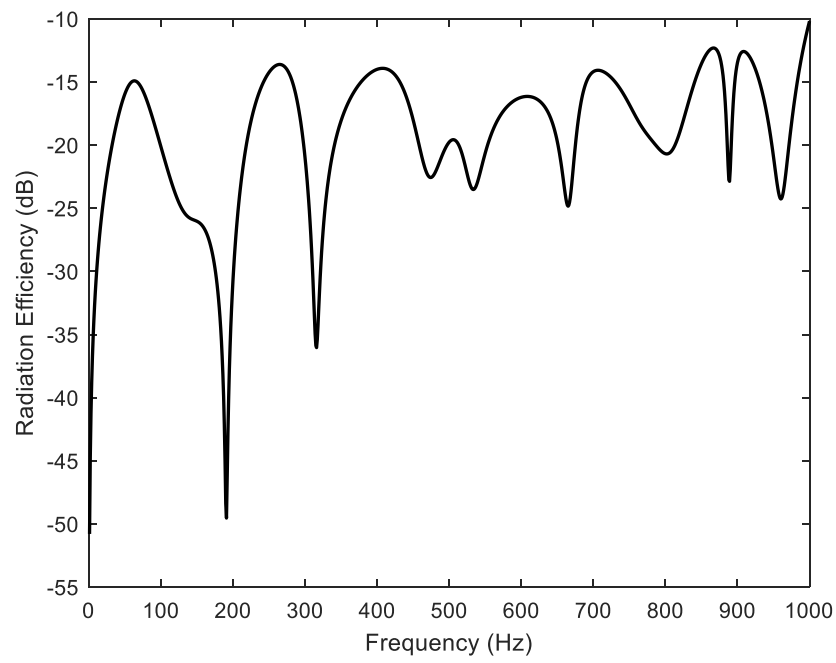

Figure 6: Radiation efficiency of the composite plate. 


\section{Conclusion}

An investigation of the vibrational and acoustical parameters of thin composite plate that is made of fibreglass has been carried out. Computational simulations have been carried out to determine deformations of the plate in square velocities for different frequency ranges for simply supported and clamped boundary conditions. It has been shown that clamping the plate at four edges delayed first resonance of the plate by $15 \mathrm{~Hz}$ and second resonance of the plate by $30 \mathrm{~Hz}$. Vibroacoustic indicators including the radiation efficiency, the mean square velocity, and the radiated sound power have been computed for a square composite plate.

\section{Acknowledgement}

This work was supported by London South Bank University Acoustic research Centre.

\section{References}

1) M. D. Waller, "Vibrations of Free Square Plates: Part I Normal Vibrating Modes", Proceedings of the Physical Society of London, Vol. 51, Jun. 1939, pp. 831-844.

2) M. D. Waller, "Vibrations of Free Square Plates: Part 11, Compounded Nom1 Modes", Proceedings of the Physical Society of London, Vol. 52, Feb. 1940, pp. 452-455.

3) G. B. Warburton, "The Vibration of Rectangular Plates", Proceedings of the Institution of Mechanical Engineers, Vol. 168, No. 12, Jan-Dec 1954, pp. 371-384.

4) R. F. S. Hearmon, "The Fundamental Frequency of Vibration of Rectangular Wood and Plywood Plates", Proceedings of the Physical Society of London, Vol. 58, Jan. 1946, pp. 78-92.

5) R. Szilard, (1974), "Theory and Analysis of Plates". "Classical and Numerical Methods". PrenticeHall, Englewood, New Jersey.

6) A. W. Leissa, "the free vibration of rectangular plates" J. Sound Vib. 1(3), 257-293(1973).

7) D. Young, "Vibration of rectangular plates by the Ritz method," Journal of Applied Mechanics 17, 448-453(1950).

8) D. D. Theodorakopoulos and D. E Beskos, "Flexural vibration of poroelastic plates," Acta Mechanica, 103, 191-203 (1994).

9) M. A. Biot, "Theory of elastic wave propagation in a fluid saturated porous solid. Part I-low frequency range," J. Acoust. Soc. Am. 28,168-178(1956).

10) M. A. Biot, "Theory of elastic wave propagation in a fluid saturated porous solid. Part 2-higher frequency range," J. Acoust. Soc. Am. 28, 179-191(1956).

11) P. Leclaire, A. Cummings and K. V. Horoshenkov, "Transverse vibration of a thin rectangular porous plate saturated by a fluid," J. Sound Vib. 247(1), 1-18(2001).

12) P. Leclaire, K. V. Horoshenkov, M. J. Swift, and D. C. Hothersall, "The vibration response of a clamped rectangular porous plate," J. Sound Vib. 217(1), 19- 31(2001).

13) P. Leclaire, "Vibration of Porous Plates in Structural Acoustic Coupling Applications," $3^{\text {rd }}$ European Congress on Acoustics. $16^{\text {th }}-20^{\text {th }}$ September 2002, Spain.

14) $\mathrm{H}$. Aygun and K. Attenborough, 2007. "Predicted effects of fluid loading on the vibration of elastic porous plates". Acta Acoustica united with Acustica. 93(2): 284-289.

15) H. Aygun and K. Attenborough, 2008, "Sound absorption by clamped poroelastic plates," J. Acoust. Soc. Am. 124 (3), 1-7. (Impact factor: 1.717), (EPSRC).

16) $\mathrm{H}$. Aygun and K. Attenborough 2008, "The insertion loss of perforated porous plates in a duct with and without mean air flow," Applied Acoustics, Vol.69, 506-513. (Impact factor: 0.887), (EPSRC).

17) H. Aygun and K. Attenborough, 2008, "Insertion loss of clamped perforated and non-perforated poroelastic plate silencers with flow," Acta Acustica united with Acustica, Vol. 94, Number 5, pp. 703-708(6). (Impact factor: 0.637), (EPSRC).

18) H. G. Davies, "Low frequency random excitation of water-loaded rectangular plates," J. Sound Vib. 15. 107-126 (1971).

19) L. D. Pope and R. C. Leibowitz, "Intermodal coupling coefficients for a fluid-loaded rectangular plate," J. Acoust. Soc. Am.56, 408-414 (1974) 
20) M.C. Gomperts, "Radiation from rigidly baffled rectangular panels with general boundary conditions," Acustica, 30, 320-327 (1974).

21) M.C. Gomperts, "Sound radiation from baffled, thin, rectangular plates," Acustica, 37, 93-102 (1977).

22) A. Berry, "A general formulation for the sound radiation from rectangular, baffled plates with arbitrary boundary conditions," J. Acoust. Soc. Am. 88(6), 2792-2802 (1990).

23) A. Berry, "A new formulation for the vibrations and sound radiation of fluid-loaded plates with elastic boundary conditions," J. Acoust. Soc. Am. 96((2), 889-901 (1994).

24) N. Atalla and J. Nicolas. "A new tool for predicting rapidly and rigorously the radiation efficiency of plate-like structures," J. Acoust. Soc. Am. 95 (6), 3369-3378(1994).

25) O. Foin, J. Nicolas, and N. Atalla, "An efficient tool for predicting the structural acoustic and vibration response of sandwich plates in light or heavy fluid," Applied Acoustics, 57, 213-242(1999).

26) B. E. Sandman, "Motion of a three-layered elastic-viscoelastic plate under fluid loading," $J$. Acoust. Soc. Am. 57(5), 1097-1107(1975).

27) H. Nelisse, O. Beslin, and J. Nicolas, "Fluid -structure coupling for an unbaffled elastic panel immersed in a diffuse field," J. Sound Vib. 198(4), 485-506(1996).

28) $\mathrm{H}$. Nelisse, O. Beslin, and J. Nicolas, "A generalized approach for the acoustic radiation from a baffled or unbaffled plate with arbitrary boundary conditions, immersed in a light or heavy fluid," $J$. Sound Vib. 211(2), 207-225(1998).

29) H. Aygun and F. McCann, 2020. "Structural and acoustical performance of recycled glass bead panels". Journal of construction and building materials. Volume 258, 119581.

30) Lord Rayleigh, "Theory of Sound" (London, 1926) Vol.(I), and (Dover, New York, 1945), Vol.(II). 